\title{
Subjective Memory Complaints and Awareness of Memory Functioning in Mild Cognitive Impairment: A Systematic Review
}

\section{J.L. Roberts L. Clare R.T. Woods}

Bangor University, Bangor, UK

\section{Key Words}

Anosognosia - Insight - Dementia - Alzheimer's disease •

Metamemory $\cdot$ Self-knowledge $\cdot$ Self-report

\begin{abstract}
Objectives: Subjective memory complaint (SMC) is central to the diagnosis of mild cognitive impairment $(\mathrm{MCl})$. People with $\mathrm{MCl}$ are at a higher risk of progressing to dementia, and research on SMC is contradictory in terms of the accuracy of SMC and its predictive role for future dementia. One possible reason for these contradictory findings is that the level of awareness of memory function may vary among people with $\mathrm{MCl}$. This review examines whether the level of awareness of memory functioning varies amongst people classified as having $\mathrm{MCl}$ and whether there is support for the suggestion that the level of awareness in $\mathrm{MCl}$ predicts future progression to dementia. Method: Sixteen studies were identified which evaluate the awareness level in people classified as having $\mathrm{MCl}$ in either a clinical or research setting. In addition to the outcome of each study, the conceptualization of awareness, 'object' of awareness and methodology were also considered. Results: There is evidence to show that the level of awareness in $\mathrm{MCl}$ does vary, and this may have implications for future progression to dementia. Conclusions: Given the increased risk of progression to dementia for those identified as having $\mathrm{MCl}$, the role of awareness
\end{abstract}

should be explored further with due consideration given to the conceptualisation of awareness and the methodology employed. The finding of variability in awareness has implications for the use of SMC in the diagnostic criteria for $\mathrm{MCl}$.

Copyright $\odot 2009$ S. Karger AG, Basel

\section{Introduction}

In mild cognitive impairment (MCI), the individual functions adequately on a day-to-day basis and has fairly intact cognitive abilities, but shows evidence of cognitive decline in a given domain or domains beyond what would be expected for his or her age [1]. Longitudinal follow-up of people with MCI shows annual conversion rates to probable Alzheimer's disease (AD) as around 10-15\%. This figure drops significantly to $1-2 \%$ per year for normal older people [2]. The importance of MCI therefore lies in its possible role as a prodromal stage of dementia. Suggested predictive factors for progression from MCI to $\mathrm{AD}$ include a pronounced decline on objective test scores in cognitive domains such as episodic memory [3-5], visual memory [4], executive function and verbal intelligence [3]. Behavioural markers, which have been suggested as predictive of progression to $\mathrm{AD}$, include depression [6] and apathy [7], and the personality variable of low levels of conscientiousness has also been implicated [8]. For

\section{KARGER}

Fax +4161306 1234 E-Mail karger@karger.ch www.karger.com (c) 2009 S. Karger AG, Basel

$1420-8008 / 09 / 0282-0095 \$ 26.00 / 0$

Accessible online at:

www.karger.com/dem
Prof. Linda Clare

School of Psychology, Bangor University

Bangor LL57 2AS (UK)

Tel. +44 1248388 178, Fax +44 1248382 599, E-Mail l.clare@bangor.ac.uk 
a review of behavioural manifestations, see Apostolova and Cummings [9]. Clearly, no definitive predictor has so far been identified.

While the term 'mild cognitive impairment' was first used by Flicker et al. [10], it was Petersen et al. [11] who first set out the diagnostic criteria for MCI as follows: (a) memory complaint, (b) intact activities of daily living, (c) intact general cognitive function, (d) memory impairment beyond what would be expected for age, and (e) no dementia. Petersen [1] went on to develop 3 subclassifications of MCI, acknowledging the heterogeneity of the construct:

- The amnestic form - memory complaint, other cognitive domains normal, not dementia.

- Multiple domain MCI - impairment in $>1$ cognitive domain, not dementia.

- Single non-memory domain MCI - isolated impairment in 1 cognitive domain, e.g. language, not dementia.

The amnestic and multiple domain criteria accord a central role to the presence of subjective memory complaint (SMC). The role of SMC was acknowledged by Petersen et al. [11] in the initial formulation of the diagnostic criteria for MCI. Later amendments $[12,13]$ suggest that any memory complaint should preferably be corroborated by an informant, although this is not a requirement. Corroboration would ensure the accuracy of the complaint, based on evidence which suggests that depression, which is highly prevalent in those with MCI [9], is a mediating factor in SMC, whereas informant accounts are more strongly correlated with objective performance. Thus, there is an implicit acknowledgment that the individual's own subjective evaluation of his/her memory functioning may not reflect an accurate appraisal of the actual memory deficit. An inaccurate appraisal could indicate a lack of awareness of changes in memory or of difficulties with memory. The key issue here is whether some individuals with memory changes amounting to MCI may lack awareness of these, and thus fail to express SMC, while meeting other aspects of the diagnostic criteria.

Different theoretical models underpin the understanding of awareness and each model defines awareness as occurring at different levels, whether it is from a neurological, psychiatric or psychosocial perspective [14] For the purpose of this review, awareness can be defined as the ability to accurately appraise aspects of one's own situation or functioning, and may be evaluated in relation to a range of domains, or 'objects', including memory [ $[15$, 16]. SMC alone does not constitute an assessment of awareness of memory function. It is the comparison of SMC with a standard such as objective testing or informant rating which represents a measure of awareness [17]. If SMCs are present, it is important to identify whether this reflects an accurate appraisal of memory functioning, suggesting good awareness, or whether the complaints are inaccurate, suggesting greater or lesser difficulty than the actual memory deficit would indicate. If SMCs are absent, this does not necessarily mean there is no memory problem; it could reflect either an absence of memory problems or an inaccurate appraisal of memory functioning that indicates poor awareness.

Awareness has been extensively researched within the field of dementia [14, 17, 18]. While the conceptualisation of awareness and the specific assessment method adopted clearly have an impact on the phenomenon that is elicited, it seems evident that the level of awareness of cognitive deficits varies considerably among individuals diagnosed with mild AD. It is of interest to consider whether such variability in awareness also arises with more mild memory difficulties and whether it is evident among individuals otherwise meeting diagnostic criteria for MCI. If this is the case, then the further question arises as to whether a low awareness in MCI could serve as a possible predictor of conversion to AD. Alternatively, if people with MCI show good awareness, then SMC could be regarded as a reliable predictor of dementia. Research on awareness in people with MCI largely focuses on SMCs, their accuracy against objective test scores and their predictive role in progression to dementia.

Results are contradictory regarding the accuracy of SMC when compared to objective test performance. Clement et al. [19] conducted a cross-sectional study to examine the nature and accuracy of SMC in MCI, AD and healthy older adults. The MCI group was selected from a memory clinic where a diagnosis of MCI was based on the Petersen et al. [12] criteria, thus requiring the presence of SMC. Correlation analysis indicated that there was a higher level of cognitive complaint in the MCI group than in the healthy older adult group, although this was restricted to certain circumstances and specific domains. There was little relationship between the domains of SMC and the nature of the actual cognitive deficits as indicated by test scores, suggesting that SMCs are based on an evaluation of general cognitive abilities rather than reflecting a precise assessment of memory performance. This in itself suggests that awareness may be compromised. However, other studies have found a strong association between SMC and objective test performance [20, 21]. 
Contradictory results can also be found in relation to the predictive role of SMC in progression to dementia. Jorm et al. [22] selected a community sample of older people and examined whether SMC predicted a change in memory test performance. The study excluded those with dementia but did not specifically identify those who potentially had MCI. Indeed, 3.7\% (28 of 721) had progressed to dementia during the time period of the study (3.6 years), implying that a proportion of the sample may already have had cognitive impairment. In this study, SMC did not predict cognitive decline, which may in itself indicate that the level of awareness was variable. As SMC was not a requirement for inclusion in the study, this sample may have included people with cognitive impairment who were unaware of any change.

Schmand et al. [23] conducted a 4-year longitudinal study of 4,051 older people living in the community, of whom 131 (6.2\%) developed dementia over the course of the study. SMC predicted conversion to dementia more strongly than age. Although the authors excluded those with dementia at the outset, as in the Jorm et al. [22] study, it is unclear which of the sample could have been classed as having MCI, thus making these results difficult to interpret. Geerlings et al. [24] used the same sample of volunteers to explore the predictive role of SMC. Based on the Mini Mental State Examination [MMSE, 25], the sample subjects were classed as either cognitively normal ( $n=1,956$ of 2,169 who were available for follow-up) or as having borderline/impaired cognition $(n=213)$. Those classed as having normal cognition had scores between 26 and 30 on the MMSE whereas those with scores $\leq 25$ were classed as borderline/impaired. An association was found between memory complaint and incident $\mathrm{AD}$, which was modified by cognition. On further analysis, memory complaints were associated with incident $\mathrm{AD}$ for those with normal cognition but not for those with borderline/impaired cognition.

The role of affective states when considering the accuracy of SMC and any predictive role in progression to dementia should not be overlooked. Minett et al. [26] found a stronger association between depressive symptoms and SMC than between poor cognitive performance and SMC, suggesting that SMCs are more likely to reflect depression than an accurate appraisal of memory. Sarazin et al. [27] assessed a total of 251 participants from a clinical population who met diagnostic criteria for MCI at 6-month intervals over a period of 3 years. Spontaneous memory complaints were found to be slightly lower in participants who converted to dementia than in those who remained stable. The authors also found no signifi- cant differences between groups in anxiety or depression scores, suggesting that although affective states may influence SMC, they are not predictive of progression to dementia.

Differences in the outcomes of these studies could be accounted for by conceptual and methodological issues similar to those found in the research on awareness in dementia [17]. Different methods are used across studies to elicit SMC, ranging from a battery of measures to a single question, and methods of objectively testing cognition also differ amongst studies. Depression, anxiety and certain personality traits are also associated with SMC and may well account for inconsistencies in the results [28]. The heterogeneity of the MCI criteria and the sampling methods used clearly impact the outcome; in particular, by only including those participants with SMCs, any individuals who are not aware of their memory difficulties will be missed. Within community samples, there may be no clear demarcation between those who are considered cognitively healthy and those who would fit into the MCI criteria.

In summary, there is evidence to suggest that the ability to accurately appraise aspects of one's own functioning, and in particular memory functioning, may be affected in some people who otherwise meet diagnostic criteria for MCI. This has implications for the emphasis placed on SMC in diagnostic criteria. It may also have implications for the likelihood of conversion to dementia, such that lower levels of awareness are predictive of progression. This review will systematically examine quantitative studies providing information about awareness in MCI in order to determine the nature of awareness in $\mathrm{MCI}$ and its role in progression to dementia. For the purposes of the review, awareness will be defined as the ability to accurately appraise one's own functioning in a given domain. The focus here will primarily be on awareness of memory functioning, although other aspects of awareness will be considered where information is available, in order to provide a broader picture. The following specific questions will be addressed:

(1) Does level of awareness of memory functioning vary among people diagnosed with MCI?

(2) Do lower levels of awareness of memory functioning in people diagnosed with MCI predict conversion to dementia? 


\section{Method}

Searches of PsychInfo, Medline, Science Direct, Web of Knowledge and Ovid were conducted on the 9th September 2008 using the search terms 'mild cognitive impairment' and 'cognitive impairment' combined with 'awareness', 'anosognosia', 'metamemory', 'insight', 'self-knowledge', 'self-report', 'evaluation', 'experience', 'memory complaints' or 'knowing'. Further studies were identified from the references cited in each selected study and examined for suitability. Criteria for inclusion in the review were:

(a) the study considered awareness in people with a diagnosis of mild cognitive impairment; and

(b) the study measured participants' awareness of memory functioning and/or other aspects of awareness.

There was no limit on the year of publication, although given that the term MCI was not used prior to 1991, it was not expected that there would be studies dated earlier than this date.

\section{Results}

Twenty-three studies were identified as potentially relevant and examined in detail. Of these, 16 studies met the inclusion criteria for the review. These studies are summarised in table 1. Firstly, methodological and conceptual issues will be considered in relation to participant selection, the concepts and definitions employed, and the aspects of awareness elicited. The evidence regarding inter-individual variability in awareness and the possible role of awareness as a predictor of conversion to dementia will then be evaluated.

\section{Methodological and Conceptual Issues}

\section{Participant Selection}

MCI was either a construct applied to a group of healthy volunteers from the community [e.g. 29-31], or a diagnosis made in a clinical setting [e.g. 32-34]. In the clinical samples, mean MMSE scores ranged from 25.9 [33] to 28.38 [35] with an average mean across studies of 27.2. Ready et al. [36] based their results on a mixed sample of people with $\mathrm{AD}$ and MCI, and MMSE data was not provided separately for the MCI group. Four studies recruited participants from the community, with only 2 reporting MMSE scores for the MCI groups; the mean MMSE scores were 26.63 (SD 1.82) [29] and 26.1 (SD 2.0) [30]. Purser et al. [37] did not use MMSE and Farias et al. [31] did not report overall MMSE scores for their MCI group. Thus, for those studies where data is available, there does not appear to be any major difference in cognitive status arising from the sampling method employed.
Numbers of participants ranged from 16 [29] to 107 [38]. Purser et al. [37] used an epidemiological database and extracted data for 2,924 individuals. The average age across MCI participants was 73.45 years (range 67.45-78; SD 3.93).

Methods for diagnosing MCI differed amongst studies, but the most frequently-used criteria were those of Petersen et al. $[12,13]$. Table 2 provides an overview of the diagnostic criteria employed in each study.

\section{Concepts and Definitions}

Awareness is a multidimensional concept with no single clear conceptual and theoretical model or definition. This is reflected in the different terms used and can be explained to some extent by the range of disciplines working towards an understanding of awareness, in relation to their epistemological foundations. The one study which did use the term 'awareness', Okonkwo et al. [35], does not offer a definition for the term. Other studies employed different terminology.

Several studies use the term 'insight'. Cook and Marsiske [29, p. 413] describe insight as the 'ability to elaborate on the experience of a disease, label the symptoms of the disease as pathological .... Ready et al. [36, p. 242] conceptualize insight as 'awareness of situation, memory deficit, functional deficits and disease progression', which merely defines insight as awareness. Crowe et al. [30] refer to insight in their conclusion, but do not offer a definition of the concept. Based on research on insight in psychosis, Onor et al. [39] identified 2 types of insight, emotional and cognitive. The authors describe emotional insight as the psychological response to the illness whereas cognitive insight is described as the recognition of symptoms without reference to psychological and emotional influence. The authors acknowledge that awareness is complex and multifaceted, and refer to literature which contradicts their definition e.g. Kessler and Supprian [40] who acknowledge that awareness influences both behavioural and cognitive functions. Such contradiction highlights the difficulties in conceptualising such a broad concept, adding little to the existing literature on the subject.

The term 'metamemory' is used by Marri et al. [41] to describe a general knowledge of cognitive functioning and processes. Perrotin et al. [42] also use the term metamemory in relation to 'metamemory judgments' which are an evaluation of performance made whilst engaging in a specific memory task. Although both studies refer to 'metamemory', there is little similarity in their definitions and this is reflected in the different methods and focus of both studies. 
The term 'anosognosia' is used by Kalbe et al. [34] to refer to unawareness of cognitive dysfunction. Ries et al. [43] use the term anosognosia to refer to unawareness of loss of function, specifically in the area of cognition. While these studies share similar conceptualisations of anosognosia and describe this as a lack of awareness, their methodology and focus differ and their conclusions on awareness in MCI are conflicting. Vogel et al. [44] use anosognosia synonymously with unawareness of deficits and lack of insight.

The studies in this review employ a range of different terms to describe the phenomenon of interest, and even where the same term is used, the meaning assigned to it is often variable. Different terms may be used interchangeably. In some instances, definitions are lacking, while in other studies a definition is provided but is contradicted by other cited material. This heterogeneity and lack of clarity necessarily results in studies with a range of different outcomes.

\section{'Objects' of Awareness}

Awareness has to be understood in relation to something. This is termed the 'object' of awareness. Awareness is expressed in relation to a given object, and the selection of the object of awareness to be investigated influences the nature of the awareness phenomenon that is elicited clinically [15]. Objects can range from the broad, such as awareness of having an illness, to the specific, such as awareness of having memory impairment or making an error on a memory task. The object of awareness may be cognitive or behavioural in nature or may be an affective state, such as apathy. The implications of this are that the phenomenon of awareness which is elicited will not be comparable across objects as these reflect different aspects of a wider awareness concept. The studies included in this review focussed on a range of objects including memory, general cognitive dysfunction, everyday functional abilities and apathy.

Seven studies assessed awareness in relation to memory functioning. Cook and Marsiske [29] compared participants' subjective memory beliefs against neuropsychological testing as a representation of awareness of memory function. They specifically focussed on subjective memory beliefs rather than complaints, as they claimed memory beliefs were better predictors of memory performance. Hanyu et al. [32] investigated unawareness of memory impairment in order to test the utility of SMC as a diagnostic entity, and later investigated [33] the correlation between awareness of memory deficit and cerebral perfusion. In a prospective cohort study, Purser et al. [37] compared the self-ratings of participants with and without SMC against objective test scores in order to determine the accuracy of SMC, thus providing an indication of level of awareness. This study specifically considered the predictive utility of SMC in predicting decline in functional disability, word recall and general cognition over a 10-year period. Vogel et al. [44] focussed on awareness of memory impairment in participants with MCI, mild $\mathrm{AD}$ and healthy controls in order to examine the nature of awareness in these groups. Using the same prospective memory clinic cohort, Vogel et al. [45] examined correlations between awareness of memory function and behavioural symptoms, executive test performance and regional cerebral blood flow in the frontal cortex.

Marri et al. [41] used a metamemory questionnaire to measure participants' beliefs about memory in everyday life, and explored correlations with measures of self-perceived health and memory test performance. In this case it is the relationship between responses on the metamemory measure and scores on the memory test that constitute a measure of awareness. Perrotin et al. [42] used an experimental metamemory paradigm, eliciting feeling of knowing (FOK) judgments as a means of evaluating the accuracy of participants' estimation of their memory performance whilst engaged in a memory task.

Four studies used functional abilities as the object of awareness. Onor et al. [39] compared perceptions held by people with MCI and mild AD regarding cognitive difficulties, functional problems and behavioural disturbances. These perceptions were also compared to those of caregivers. Farias et al. [31] focussed on the lack of agreement between people with dementia and their caregivers regarding cognitive and functional abilities, and examined this phenomenon in participants classified as having MCI and dementia. Tabert et al. [38] specifically focussed on functional deficits, again comparing self- and informant reports of functional deficits in people identified as having MCI and AD. Okonkwo et al. [35] focussed on financial ability as the object of awareness.

Three studies assessed awareness of cognitive function through the use of questionnaires covering various cognitive domains. Crowe et al. [30] hypothesised that poorer subjective cognitive function at baseline would predict future decline over the 2-year period of the study, comparing subjective cognitive function with a measure of global cognitive status at baseline and subsequent follow up. Two studies focussed on the awareness of cognitive dysfunction. Kalbe et al. [34] compared the level of awareness of cognitive dysfunction to a global measure 


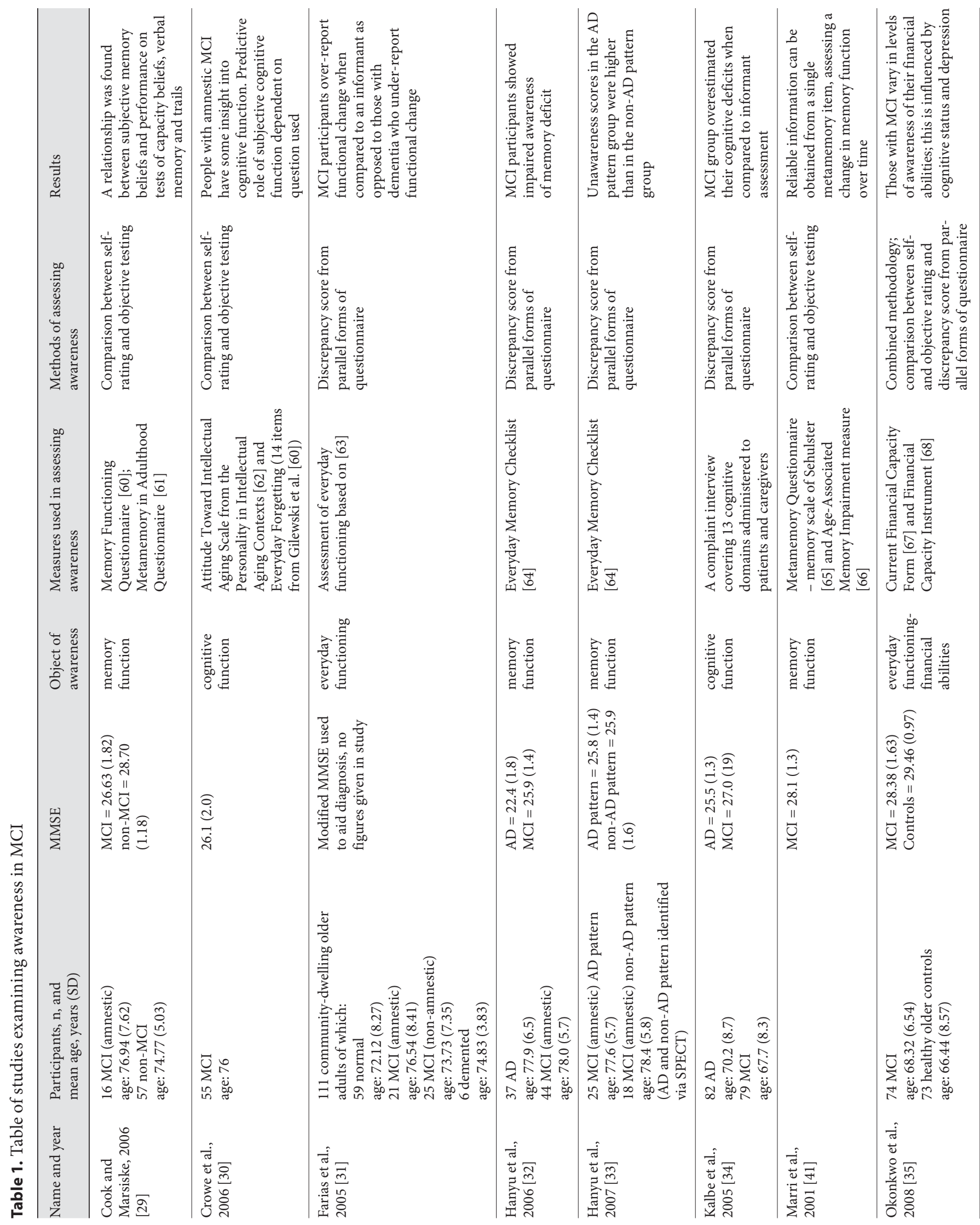




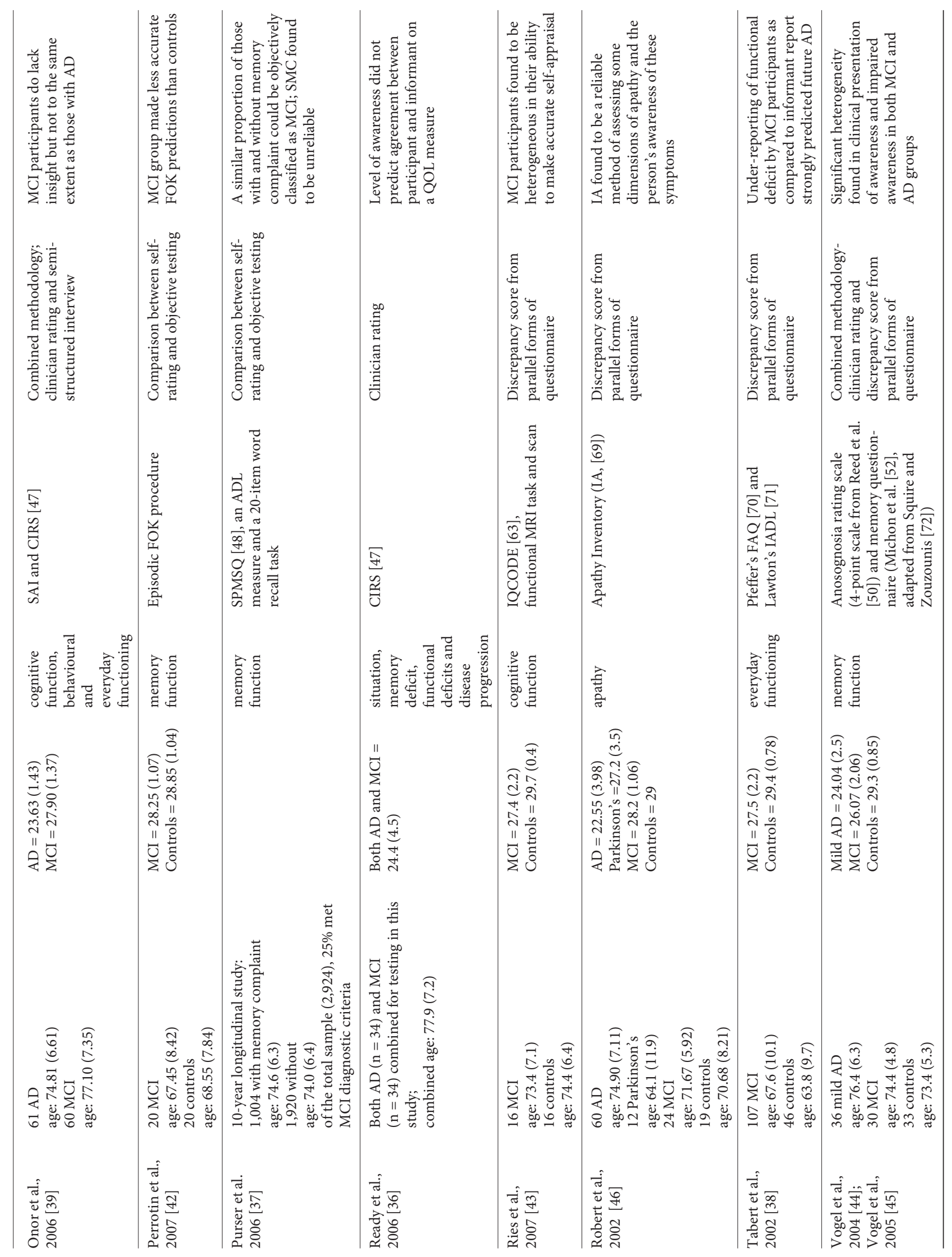


Table 2. MCI diagnostic criteria used in reviewed studies

\begin{tabular}{|c|c|c|c|c|c|c|c|c|}
\hline & $\begin{array}{l}\text { Winblad } \\
\text { (2004) } \\
{[73]}\end{array}$ & $\begin{array}{l}\text { Petersen } \\
\text { et al. } \\
(2001) \\
{[13]}\end{array}$ & $\begin{array}{l}\text { Petersen } \\
\text { et al. } \\
(2001) \\
{[13]^{1}}\end{array}$ & $\begin{array}{l}\text { Petersen } \\
\text { et al. } \\
(1999)^{2} \\
{[12]}\end{array}$ & $\begin{array}{l}\text { Petersen } \\
\text { et al. } \\
(1999)^{3} \\
{[12]}\end{array}$ & $\begin{array}{l}\text { Below 10th } \\
\text { percentile } \\
\text { on verbal } \\
\text { memory }\end{array}$ & $\begin{array}{l}\text { Below 10th } \\
\text { percentile on } \\
\text { non-memory } \\
\text { measures }\end{array}$ & $\begin{array}{l}\text { Deficits in neuropsycho- } \\
\text { logical testing and/or } \\
\text { memory complaint and } \\
\text { functional impairment }\end{array}$ \\
\hline Cook and Marsiske (2006) [29] & & & $\sqrt{ }$ & & & & & \\
\hline Crowe et al. (2006) [30] & & & & & $\checkmark$ & & & \\
\hline Farias et al. (2005) [31] & & & & & & $\sqrt{ }$ & $\sqrt{ }$ & \\
\hline Hanyu et al. (2006) [32] & & $\sqrt{ }$ & & & & & & \\
\hline Hanyu et al. (2007) [33] & & $\sqrt{ }$ & & & & & & \\
\hline Kalbe et al. (2005) [34] & & & & $\sqrt{ }$ & & & & \\
\hline Marri et al. (2001) [41] & & & & $\sqrt{ }$ & & & & \\
\hline Okonkwo et al. (2008) [35] & & $\sqrt{ }$ & & & & & & \\
\hline Onor et al. (2006) [39] & & $\sqrt{ }$ & & & & & & \\
\hline Perrotin et al. (2007) [42] & & $\sqrt{ }$ & & & & & & \\
\hline Purser et al. (2006) [37] & & & & $\sqrt{ }$ & & & & \\
\hline Ready et al. (2006) [36] & & $\sqrt{ }$ & & & & & & \\
\hline Ries et al. (2007) [43] & $\sqrt{ }$ & & & & & & & \\
\hline \multicolumn{9}{|l|}{ Robert et al. (2002) [46] } \\
\hline Tabert et al. (2002) [38] & & & & & & & & $\checkmark$ \\
\hline Vogel et al. $(2004,2005)[44,45]$ & & $\sqrt{ }$ & & & & & & \\
\hline
\end{tabular}

of cognitive status; while Ries et al. [43] compared the level of awareness of cognitive dysfunction to the brain pathology of participants engaged in a functional MRI task involving self-appraisal (the participants selected trait adjectives which related to them). This particular study highlights the difficulty inherent in the comparison of different objects of awareness. Awareness of cognitive dysfunction, based on informant and participant rating of change over a 10-year period, bears little relationship to self-appraisal of ability on an experimental task. Each specific awareness measure will elicit a different awareness phenomenon, rather than a global indication of awareness.

Ready et al. [36] examined the relationship between awareness level and the reliability and validity of self-reported quality of life from people with $\mathrm{MCI}$ and $\mathrm{AD}$, as compared to informant rating of the participant's quality of life. They used a clinician rating of awareness where the objects were awareness of situation, memory deficit, functional deficits and disease progression. Again, there is little relationship between clinician rating of awareness in cognitive domains and the subjective response to questions about quality of life.
Robert et al. [46] examined awareness of apathy in order to establish the reliability and validity of the Apathy Rating scale. A questionnaire assessing emotional, behavioural and cognitive dimensions of apathy was administered to the participant and informant, the awareness of these symptoms of apathy being the object of awareness. A comparison was made between participants with AD, MCI and Parkinson's disease. The relationship between awareness and apathy is complex, in that the symptoms of apathy can directly affect a person's expressions of awareness. If an individual is apathetic, this is likely to be reflected in his/her responses on the questionnaire. This does not, however, indicate that the person is necessarily unaware.

What is clear from these studies is that the chosen object of awareness directly influences the nature of the phenomenon that is elicited. The variation in selection of objects makes it difficult to draw comparisons across studies. Even where the object is the same, different research questions and the use of different methods to assess awareness create difficulties in making comparisons. 


\section{Measurement of Awareness}

Clare [14] identified 3 main methodological approaches to measuring awareness in $\mathrm{AD}$ that are typically used either singly or in combination:

- Awareness rating by a clinician.

- Discrepancy score between self- and informant rating on parallel forms of questionnaires.

- Discrepancy score between self-rating and actual performance on an objective task.

The same methods of assessing awareness were found in the studies of MCI included in this review. Each method will be discussed in terms of its advantages and limitations.

Only Ready et al. [36] relied on clinician rating alone. The clinician rated the participants according to a 4 -item scale, the clinical insight rating scale (CIRS) [47], which yields scores ranging from 0 (fully aware) to 8 (totally unaware). This scale relates to awareness of situation, memory deficit, functional deficits and disease progression. Clinician rating is subjective and relies on the participant and clinician having a reciprocal relationship, where the participant understands the questions asked and is able to respond in a way which is understandable to the clinician. It is assumed that the clinician has some knowledge of awareness and is able to assess this accurately. Clinicians will also have the advantage of talking to the individual and treating them as such, picking up subtle cues, whereas questionnaires will not. However, the patients may present themselves to a clinician in a certain way which may not reflect the reality of their situation.

Seven studies used the discrepancy between self- and informant rating on parallel questionnaires as a measure of awareness. In some cases, validated questionnaires were used [e.g. 32, 33] but other studies used non-validated measures [e.g. 34]. These questionnaires were selected with regard to the chosen object of awareness. It is important to note that the method of questioning used is likely to have an impact on the kind of responses that are elicited [15]. The questionnaire discrepancy method also assumes that the informant is accurately assessing the abilities of the person with MCI, which may not necessarily be the case. Ries et al. [43] acknowledge this limitation, noting that even those informants who have known the person with MCI for many years may tend to over- or under-report symptoms or are unaware of the extent of cognitive decline.

Five studies used a comparison between self-rating and performance on objective testing as a measure of awareness. These studies focussed on subjective memory complaints or memory beliefs $[29,30,37]$, or metamem- ory $[41,42]$. Each of these studies used questionnaires to elicit a self-rating of memory [e.g. 29] or cognitive function [e.g. 30]. The objective testing ranged from a broad, global test of cognitive function such as the MMSE [29, 30], to a more comprehensive memory test such as the Randt memory test used by Marri et al. [41].

Purser et al. [37] used data from an epidemiological database to form a prospective cohort study over a 10 year period. The MCI group was split between those with and those without memory complaints and a comparison was made with objective scores on the 7-item Short Portable Mental Status Questionnaire [SPMSQ, 48], an ADL measure and a 20 -item word recall task. The nature of the specific memory complaints is not described, and it is questionable to what extent these complaints are related to the objective items tested. For example, a 20 -item word recall task would not necessarily reflect the problems an individual may have in everyday situations. If awareness is measured by the level of agreement between the selfrating and objective test, there should be a similar focus between the two in order to assess awareness correctly, thus highlighting a need for isomorphic measures [17]. Perrotin et al. [42] acknowledge the need for similarly focussed measures based on difficulties they encountered in comparing SMC with a specific experimental paradigm. Their results show that their MCI participants had a higher level of SMC compared to healthy controls, whereas the MCI group over-estimated their predicted performance on the experimental task. They provide various possible explanations for these results but acknowledge the methodological limitations of using such divergent measures.

Three studies used a combined methodology. Clinician rating on the clinical insight rating scale was used by Onor et al. [39] together with the Schedule for the Assessment of Insight (SAI) [49], a semi-structured interview scale developed for measuring awareness in psychosis. Onor and colleagues acknowledge that the SAI is not ideal, given that the questions relate to symptoms of psychosis. Having a combined methodology offers the possibility of cross-validating different methods of assessing awareness, but given that the SAI is more suited to psychotic conditions it is unclear why this particular scale was chosen for studying participants with MCI.

Vogel et al. [44] used discrepancy scores on parallel forms of a memory questionnaire and clinician rating of awareness on the Anosognosia Rating Scale [50]. This is a 4-category scale ('full awareness', 'shallow awareness', 'no awareness', 'denies impairment'). Vogel et al. [44], acknowledging the lack of a 'gold standard' in awareness 
rating, compared clinician ratings with discrepancy scores. The awareness rating based on discrepancy scores between participant and informant on a memory questionnaire corresponded well with clinician ratings, providing support that they both measure similar things. Although the authors conclude that in a clinical situation this provides evidence for the accuracy of the clinician rating of awareness, they acknowledge the need for multiple methods of assessing awareness in research situations.

Okonkwo et al. [35] used a discrepancy score between participant and informant on a parallel measure of financial capacity in addition to a performance-based psychometric instrument for assessing the financial ability of older adults. This allowed the authors to compare the participant self-report with a performance-based measure in addition to assessing the accuracy of informant report against objective testing. The performance-based measure in this study was developed using the same conceptual model as the measure of financial ability, thus ensuring isomorphism. The performance-based task was also something which was familiar to the participant. The development of methods which are isomorphic and use familiar situations are recommended [17].

In the studies included in this review, a variety of methods were used to measure awareness. The choice of method is influenced to some extent by the conceptualisation of awareness and the selected object of awareness. There are issues relating to the subjectivity of clinician ratings, the accuracy of informant ratings and the comparability of subjective and objective rating measures. Although a combined methodology may appear preferable, this may add further complications if the limitations inherent in each method are not acknowledged and taken into account.

\section{Evidence for Individual Variability in Awareness of Memory Functioning in MCI}

Despite the limitations outlined above, the studies included in this review provide evidence to suggest that people diagnosed with MCI differ in level of awareness, and that some individuals with MCI have low levels of awareness. In general, some participants with MCI did show reduced awareness of memory impairment, but not to the same extent as people with dementia [e.g. 32]. Hanyu et al. [33] used single photon emission computed tomography (SPECT) to identify MCI participants with reduced cerebral perfusion in bilateral parietotemporal or posterior cingulate areas who were defined as having an $\mathrm{AD}$ pattern brain pathology. The group showing the AD pattern brain pathology had lower levels of awareness than the non-AD pattern group.

Onor et al. [39] found that informants reported more cognitive and behavioural impairment than did individuals with MCI, suggesting a reduced awareness among people with MCI. Vogel et al. [44] found that people with MCI showed similar levels of impaired awareness to people with mild $\mathrm{AD}$. Within both $\mathrm{MCI}$ and $\mathrm{AD}$ groups, however, there was a full spectrum of awareness, with some participants showing good awareness and others poor awareness. Some of the participants with MCI showed a significant lack of awareness.

Purser et al. [37], in a sample including people with and without SMC, focussed on the validity of SMCs and found these to be an unreliable measure of cognitive impairment. This implies inaccurate judgments, and thus supports the idea that level of awareness is variable in MCI. Crowe et al. [30], who did not use presence of SMC as a criterion for inclusion in their MCI group, also included people with and without SMC. In this study, the MCI group showed some awareness regarding memory and cognitive difficulties, with measures of change in cognitive function and a single question about change in memory predicting significant change in MMSE scores at 2-year follow-up. Questions relating to frequency of problems with memory in everyday situations did not predict significant change. The authors conclude that asking about 'change' is preferable to asking about 'problems', given that a participant may be aware of a decline in memory but not perceive this as a problem.

Kalbe et al. [34] suggest that low awareness as measured by a discrepancy score between participant and informant is a frequent symptom of $\mathrm{AD}$ but not MCI. However, their results also indicate that people with MCI demonstrate low awareness. With regard to their discrepancy scores, positive values reflected unawareness whilst negative values implied good awareness. Discrepancy scores were not always negative for people with MCI (median $=-1$, range -19 to 12 ). This range of scores suggests that level of awareness varies, with some participants showing limited awareness and others reporting significantly more dysfunction than their informants. In this study, SMC was required for the MCI diagnosis, so that people who did not complain of memory dysfunction but did have impairments may have been excluded.

Over-estimation of dysfunction is also found in the samples reported by Farias et al. [31], Tabert et al. [38] and Okonkwo et al. [35]. Farias et al. suggest that people with 
MCI are fairly accurate in reporting their functional status, as there were few discrepancies between participants and informants. However, they acknowledge that informant- and participant-reported functional change becomes more discrepant in dementia and note that their community sample may have shown less functional change than would be found in a clinical sample [e.g. 38] Despite using different measures of functional ability, both studies found that the MCI group over-reported functional change compared to informants. Okonkwo et al. [35] found that those who over-reported dysfunction in financial ability when compared to results from an objective measure of performance had higher levels of depressive symptoms.

Although it is difficult to generalise given the incomparability across studies, there is evidence to suggest that awareness in MCI is individually variable, with some suggestion that a proportion of people with MCI may tend on average to over-estimate dysfunction. This apparent over-estimation of dysfunction may be a result of disparity between participant and informant, where the informant does not acknowledge the participant's difficulties, or the participant may be influenced by factors other than awareness when faced with an objective measure. This warrants further investigation in order to establish the factors involved in these inaccurate judgments and how these factors influence the elicited awareness phenomenon.

\section{Evidence for Low Awareness as a Predictor Variable in Conversion to Dementia}

Only a single study directly considered the role of awareness in progression from MCI to dementia. Tabert et al. [38] found strong evidence to support the view that low awareness of functional deficits in MCI predicts future dementia. Specifically, under-reporting of functional deficits compared to informant reports strongly predicted future progression to dementia. Tabert and colleagues evaluated 107 participants with MCI at baseline, 23 of whom were incident dementia cases at follow-up (mean duration 24.5 months, SD 14.3 months). Informants for those participants who converted to dementia reported greater deficits than informants for those who did not.

Not every person with a diagnosis of MCI will convert to dementia, but for those who do, the decline in functional ability would become evident at the point of progression. Indeed, it is the lack of functional deficits which is often a key discriminator in the decision to assign an MCI rather than dementia diagnosis. Tabert et al. [38] identified 2 groups of MCI participants, those with a Clinical Dementia Rating (CDR) [51] status of $0(n=39)$ and those with a CDR status of $0.5(n=53)$. The higher CDR would be thought of by many as moving into questionable dementia territory, and thus, one would expect a higher rate of progression. It is unclear, however, whether the participants with higher CDRs were more likely to have converted. Petersen et al. [12] highlight the difficulty with the heterogeneity of a $0.5 \mathrm{CDR}$, and suggest that those falling into this category that remain within the MCI classification would have significant memory impairment but retain fairly normal cognition in other areas and would only be slightly affected in activities of daily living. They suggest that those with functional impairment would be distinguishable to clinicians as having $\mathrm{AD}$.

Findings regarding the role of low awareness in progression from MCI to dementia are limited to 1 study, where the object of awareness is functional ability. The issue here is the demarcation between an MCI diagnosis and probable dementia. If an individual with MCI is to progress to dementia, decline in functional ability will occur at some point along the continuum of the disease. However, it is also probable that many of those with an MCI diagnosis may have some degree of functional difficulty $[31,38]$, which may or may not be a result of cognitive impairment. The findings of the Tabert study should not be discounted on this basis alone. Indeed, this study highlights the importance of examining whether low levels of awareness in MCI do predict future conversion to dementia and should be a focus of future research in the field.

\section{Discussion}

The overall questions of this review were firstly whether level of awareness varies among people diagnosed with $\mathrm{MCI}$, and secondly whether low levels of awareness in people with MCI predict conversion to dementia.

This review has found strong evidence for variability in the level of awareness among individuals with MCI. The included studies indicated that some individuals with MCI have limited awareness. There was also evidence to show that some individuals overestimate their dysfunction, which can be viewed as reflecting heightened or even hyper-awareness. Such over-estimation of deficits can also be found in people with $\mathrm{AD}$ [e.g. 52]. As 
regards the role of awareness as a predictor of progression, however, there is an absence of evidence. Although Tabert et al. [38] found that unawareness of functional deficit predicted future progression to dementia, methodological issues may have affected the reliability of this finding, and further studies are required in this area. The fact that the level of awareness in MCI has been found to vary in this review supports the idea that its role in the progression from MCI to dementia should be explored further. Future studies would need to examine the profile of awareness in MCI that is most indicative of progression to dementia.

The conclusions that can be drawn from the present review are limited given the lack of comparability between the included studies. A range of theoretical foundations underpinning the study of awareness are reflected in the range of terms used and the contradictory explanations offered. The broad nature and complexity of awareness is acknowledged by some although not by all. SMC, when compared to some kind of standard such as objective testing, reflects the accuracy of complaint and is therefore a measure of awareness, but excludes those who may have memory problems but who are not aware. Future research should consider focussing on the awareness level rather than SMC in order to address this issue. It is clear that further work is required on the theoretical conceptualisation of awareness in order to ensure the comparability of future studies.

The choice of the object of awareness to be assessed influences the nature of the awareness phenomenon elicited. The objects chosen by the studies in this review include both cognitive domains, for example, memory or cognitive dysfunction, and behavioural domains, for example, apathy or everyday functional abilities. Issues relating to the measurement of awareness in MCI are similar to those discussed in the dementia literature and future research should seek to address these issues through the development of new methods. These could include taking account of participant and informant factors which influence results, the use of isomorphic rating scales with objective tasks, and the use of familiar situations when examining functional ability. Studies could be improved with clearer descriptions of sample characteristics and selection procedure and additional approaches could be considered when examining awareness [14]. Given that MCI may precede the onset of dementia, there is a need for homogeneity of research methods when examining factors relative to both conditions.

Affective states can be problematic when considering awareness. Depression is common in people with MCI
[9]. Kumar et al. [53] found depression to be a predictor of MCI with motivation-related symptoms being significant predictors. They suggest that depression may mistakenly be diagnosed in those exhibiting symptoms of apathy. If unmotivated, subjective opinion may well be compromised and the relationship between SMC and depression should therefore be considered. Schmand et al. [23] found that the relationship between SMC and depression is reciprocal, where one would expect an increase in complaints about memory as a result of depression and where recognition of failing memory could lead to depression. This has implications for the study of awareness, as expressions of awareness may well be influenced by depression. This could account for over-reporting of functional difficulty as compared to objective testing or informant report which is evident in some of the studies included in this review. The presence of depression will enhance negative attributions, thus making the memory problems seem worse than they are.

It is also important to consider the psychological impact of living with MCI rather than having a diagnosis of dementia. Awareness is likely to be affected by psychological factors as well as neurological causes, since receiving such a diagnosis may directly impact on the individual's expression of awareness. Lingler et al. [54] studied the impact of living with MCI over a 6-month period, using a grounded theory approach. Emotional reactions included distress and anger, relief at the absence of a dementia diagnosis, satisfaction at having the cognitive impairment objectively verified and acceptance of the condition. The authors acknowledge the potential role of awareness in accounting for why some individuals did not acknowledge the possibility that MCI could progress to dementia, while others believed MCI would inevitably result in dementia. The label of MCI is vague and does not indicate to the person receiving the diagnosis whether it is a disease entity or part of normal aging [55]. The effect of an MCI diagnosis on the individual requires further exploration, given that the term MCI is not known to many [56] and lack of information accompanying the diagnosis may impact on coping responses [57]. This could lead to expressions of unawareness which result from confusion about MCI rather than from a person's appraisal of his/her memory.

Alongside the complexity surrounding the study of awareness, there are also complex issues relating to the construct of MCI. This is demonstrated by the range of diagnostic criteria adopted by studies in this review. Although the diagnostic criteria proposed by Petersen et al. $[12,13]$ dominate, there are differences in the use of these 
criteria, highlighting issues relating to interpretation. Alladi et al. [58] examined the applicability of research criteria for MCI in a memory clinic population and concluded that MCI case definition is highly dependent upon the neuropsychological tests used and parameters applied. Thompson and Hodges [59] conclude that MCI is a clinically useful concept, but identify 3 areas which require clarification in research settings:

(1) Consistency of tests used to identify memory impairment and the threshold used.

(2)The exclusion of non-amnestic deficits in some cases.

(3) The need for better methods of assessing functional impairment and clarification as to the nature of functional impairment in MCI.

These issues contribute to the lack of comparability across studies in this review.

\section{Conclusions}

This review has demonstrated that level of awareness varies amongst individuals diagnosed with MCI. Further work is required to determine whether low awareness is predictive of future progression to dementia, given the limited evidence in this area. There is some acknowledgment in the literature of the complexity surrounding the awareness concept, although further clarification is required. In addition to the conceptual and measurement issues already raised, future work could usefully acknowledge both neurological and psychological aspects of awareness. As the construct of MCI may be variably defined, a global set of criteria would help to ensure consistency of diagnosis amongst researchers and clinicians. Such action may lead to better understanding of the nature of SMC and awareness in MCI, which could ultimately result in better outcomes for clinicians, researchers and people affected by MCI.

\section{Acknowledgements}

J.L. Roberts was supported by an ESRC PhD studentship linked to ESRC grant RES-062-23-0371 awarded to L. Clare (PI), I.S. Marková, R.G. Morris, I. Roth and R.T. Woods.

\section{References}

1 Petersen RC: Mild Cognitive Impairment: aging to Alzheimer's Disease. New York, Oxford University Press, 2004

$\checkmark 2$ Petersen RC: Mild cognitive impairment: transition between aging and Alzheimer's disease. Neurologia 2000;15:93-101.

-3 Grober E, Hall CB, Lipton RB, Zonderman AB, Resnick SM, Kawas C: Memory impairment, executive dysfunction, and intellectual decline in preclinical Alzheimer's disease. J Int Neuropsychol Soc 2008;14:266-278.

4 Guarch J, Marcos T, Salamero M, Gastó C, Blesa R: Mild cognitive impairment: a risk indicator of later dementia, or a preclinical phase of the disease? Int J Geriatr Psychiatry 2008;23:257-265.

5 Perri R, Serra L, Carlesimo GA, Caltagirone $\mathrm{C}$ : Amnestic mild cognitive impairment: difference of memory profile in subjects who converted or did not convert to Alzheimer's disease. Neuropsychology 2007;21:549-558.

-6 Gabryelewicz T, Styczynska M, Luczywek E, Barczak A, Pfeffer A, Androsiuk W, et al: The rate of conversion of mild cognitive impairment to dementia: predictive role of depression. Int J Geriatr Psychiatry 2007;22:563567.
7 Robert PH, Berr C, Volteau M, Bertogliati C, Benoit M, Sarazin M, Legrain S, Dubois B: Apathy in patients with mild cognitive impairment and the risk of developing dementia of Alzheimer's disease: a one year follow up study. Clin Neurol Neurosurg 2006;108. 733-736.

8 Wilson RS, Schneider JA, Arnold SE, Bienias JL, Bennet DA: Conscientiousness and the incidence of Alzheimer's disease and mild cognitive impairment. Arch Gen Psychiatry 2007;64:1204-1212.

-9 Apostolova LG, Cummings JL: Neuropsychiatric manifestations in mild cognitive impairment: a systematic review of the literature. Dement Geriatr Cogn Disord 2008;25: 115-126.

10 Flicker C, Ferris S, Reisberg B: Mild cognitive impairment in the elderly: predictors of dementia. Neurology 1991;41:1006-1009.

- 11 Petersen RC, Smith GE, Waring SC, Ivnick RJ, Kokmen E, Tangalos EG: Aging, memory and mild cognitive impairment. Int Psychogeriatr 1997;9:65-69.

12 Petersen RC, Smith GE, Waring SC, Ivnick RJ, Tangalos EG, Kokmen E: Mild cognitive impairment - clinical characterization and outcome. Arch Neurol 1999;56:303-308.
13 Petersen RC, Stevens J, Ganguli M, Tangalos EG, Cummings JL, DeKosky ST: Practice parameter: early detection of dementia: mild cognitive impairment (an evidence-based review): report of the Quality Standards Subcommittee of the American Academy of Neurology. Neurology 2001;56:1133-1142.

14 Clare L: The construction of awareness in early-stage Alzheimer's disease: a review of concepts and models. Br J Clin Psychol 2004; 43:155-175.

15 Marková IS, Berrios GE: The 'object' of insight assessment: relationship to insight 'structure'. Psychopathology 2001;34:245252.

16 Clare L: Neuropsychological Rehabilitation and People with Dementia. Hove, Psychology Press, 2007.

17 Clare L, Markova I, Verhey F, Kenny G: Awareness in dementia: a review of assessment methods and measure. Aging Ment Health 2005;9:394-413.

18 Clare L: Awareness in early-stage Alzheimer's disease: a review of methods and evidence. Br J Clin Psychol 2004;43:177-196. 
19 Clement F, Belleville S, Gauthier S: Cognitive complaint in mild cognitive impairment and Alzheimer's disease. J Int Neuropsychol Soc 2008; 14:222-232.

20 Jonker C, Geerlings MI, Schmand B: Are memory complaints predictive for dementia? A review of clinical and population based studies. Int J Geriatr Psychiatry 2000; 15:983-991.

-21 Podewils LJ, McLay RN, Rebook GW, Lyketsos CG: Relationship of self-perceptions of memory and worry to objective measures of memory and cognition in the general population. Psychosomatics 2003;44:461-470.

-22 Jorm AF, Christensen H, Korten AE, Jacomb PA, Mackinnon A: Do cognitive complaints either predict future cognitive decline or reflect past cognitive decline? A longitudinal study of an elderly community sample. Psychol Med 1997;27:91-98.

-23 Schmand B, Jonker C, Geerlings MI, Lindeboom J: Subjective memory complaints in the elderly: depressive symptoms and future dementia. Br J Psychiatry 1997;171:373-376.

-24 Geerlings MI, Jonker C, Bouter LM, Adèr HJ, Schmand B: Association between memory complaints and incident Alzheimer's disease in elderly people with normal baseline cognition. Am J Psychiatry 1999;156:531-537.

-25 Folstein MF, Folstein SE, McHugh PR: 'MiniMental State': a practical method for grading the cognitive state of patients for the clinician. J Psychiatr Res 1975;12:189-198.

-26 Minett TSC, Da Silva RV, Ortiz KZ, Bertolucci PHF: Subjective memory complaints in an elderly sample: a cross-sectional study. Int J Geriatr Psychiatry 2007;23:49-54.

-27 Sarazin M, Berr C, De Rotrou J, Fabrigoule C, Pasquier F, Legrain S, Michel B, Puel M, Volteau M, Touchon J, Verny M, Dubois B: Amnestic syndrome of the medial temporal type identifies prodromal AD: a longitudinal study. Neurology 2007;69:1859-1867.

28 Reid LM, MacLullich AMJ: Subjective memory complaints and cognitive impairment in older people. Dement Geriatr Cogn Disord 2006;22:471-485.

29 Cook S, Marsiske M: Subjective memory beliefs and cognitive performance in normal and mildly impaired older adults. Aging Ment Health 2006;10:413-423.

-30 Crowe M, Andel R, Wadley V, Cook S, Unverzagt F, Marsiske M, Ball K: Subjective cognitive function and decline among older adults with psychometrically defined amnestic MCI. Int J Geriatr Psychiatry 2006;21: 1187-1192.

- 31 Farias ST, Mungas D, Jagust W: Degree of discrepancy between self and other-reported everyday functioning by cognitive status: dementia, mild cognitive impairment, and healthy elders. Int J Geriatr Psychiatry 2005; 20:827-834.

32 Hanyu H, Sakurai H, Iwamoto T: Are subjective memory complaints mandatory for the diagnosis of mild cognitive impairment? Intern Med 2007;46:791-792.
33 Hanyu H, Sakurai H, Hirao K, Shimizu S, Iwamoto T: Unawareness of memory deficits depending on cerebral perfusion pattern in mild cognitive impairment. J Am Geriatr Soc 2007;55:470-471.

34 Kalbe E, Salmon E, Perani D, Holthoff V, Sorbi S, Elsner A, Weisenbach S, Brand M, Lenz O, Kessler J, Luedecke S, Ortelli P, Herholz K: Anosognosia in very mild Alzheimer's disease but not in mild cognitive impairment. Dement Geriatr Cogn Disord 2005;19:349-356.

35 Okonkwo OC, Wadley VG, Griffith HR, Belue $\mathrm{K}$, Lanza S, Zamrini EY, Harrell LE, Brockington JC, Clark D, Raman R, Marson DC: Awareness of deficits in financial abilities in patients with mild cognitive impairment: going beyond self-informant discrepancy. Am J Geriatr Psychiatry 2008; 16: 650-659.

36 Ready RE, Ott BR, Grace J: Insight and cognitive impairment: effects on quality-of-life reports from mild cognitive impairment and Alzheimer's disease patients. Am J Alzheimers Dis Other Demen 2006;21:242-248.

37 Purser JL, Fillenbaum GC, Wallace RB Memory complaint is not necessary for diagnosis of mild cognitive impairment and does not predict 10-year trajectories of functional disability, word recall, or short portable mental status questionnaire limitations. Am Geriatr Soc 2006;54:335-338.

38 Tabert MH, Albert SM, Borukhova-Milov L, Camacho Y, Pelton G, Liu X, Stern Y, Devanand DP: Functional deficits in patients with mild cognitive impairment: prediction of AD. Neurology 2002;58:758-764.

39 Onor ML, Trevisiol M, Negro C, Aguglia E: Different perception of cognitive impairment, behavioural disturbances and functional disabilities between persons with mild cognitive impairment and mild Alzheimer's disease and their caregivers. Am J Alzheimers Dis Other Demen 2006;21:333-338.

40 Kessler H, Supprian T: Awareness of deficits in patients with dementia of the Alzheimer's type. Fortschr Neurol Psychiatr 2003;71: 541-548.

41 Marri L, Modugno M, Iacono S, Renzetti C, De Vreese LP, Neri M: Metamemory and self-perceived health in mild cognitive impairment. Arch Gerontol Geriatr 2001;suppl 7:235-244

42 Perrotin A, Belleville S, Isingrini M: Metamemory monitoring in mild cognitive impairment: evidence of a less accurate episodic feeling-of-knowing. Neuropsychologia 2007;45:2811-2826.

43 Ries ML, Jabbar BM, Schmitz TW, Trivedi MA, Gleason CE, Carlsson CM, Rowley HA, Asthana S, Johnson SC: Anosognosia in mild cognitive impairment: relationship to activation of cortical midline structures involved in self-appraisal. J Int Neuropsychol Soc 2007; 13:450-461.
44 Vogel A, Stokholm J, Gade A, Andersen BB, Hejl A, Waldemar G: Awareness of deficits in mild cognitive impairment and Alzheimer's disease: do MCI patients have impaired insight? Dement Geriatr Cogn Disord 2004;17: 181-187.

45 Vogel A, Hasselbalch SG, Gade A, Ziebell M, Waldemar G: Cognitive and functional neuroimaging correlates for anosognosia in mild cognitive impairment and Alzheimer's disease. Int J Geriatr Psychiatry 2005;20: 238-246.

46 Robert P H, Clairet S, Benoit M, Koutaich J, Bertogliati C, Tible O, Caci $\mathrm{H}$, Borg $\mathrm{M}$, Brocker P, Bedoucha P: The Apathy Inventory: assessment of apathy and awareness in Alzheimer's disease, Parkinson's disease and mild cognitive impairment. Int J Geriatr Psychiatry 2002;17:1099-1105.

47 Ott B R, LaFleche G, Whelihan WM, Buongiorno GW, Albert MS, Fogel BS: Impaired awareness of deficits in Alzheimer's disease. Alzheimer Dis Assoc Disord 1996;10:68-67.

48 Pfeiffer E: A short portable mental status questionnaire for the assessment of organic brain deficit in elderly patients. J Am Geriatr Soc 1975;23:433-441.

49 David A, Buchanan A, Reed A: The assessment of insight in psychosis. Br J Psychiatry 1992;161:599-602.

50 Reed BR, Jagust WJ, Coulter L: Anosognosia in Alzheimer's disease: relationships to depression, cognitive function, and cerebral perfusion. J Clin Exp Neuropsychol 1993;15: 231-244.

-51 Hughes CP, Berg L, Danziger WL, Coben LA, Martin RL: A new clinical scale for the staging of dementia. Br J Psychiatry 1982; 140:566-572.

-52 Michon A, Deweer B, Pillon B, Agid Y, Dubois B: Relation of anosognosia to frontal lobe dysfunction in Alzheimer's disease. J Neurol Neurosurg Psychiatry 1994;57:805809.

53 Kumar R, Jorm AF, Parslow RA, Sachdev S: Depression in mild cognitive impairment in a community sample of individuals 60-64 years old. Int Psychogeriatr 2006;18:471480.

54 Lingler JH, Nightingale MC, Erlen JA, Kane AL, Reynolds CF, Schulz R, DeKosky ST: Making sense of mild cognitive impairment: a qualitative exploration of the patient's experience. Gerontologist 2006;46:791-800

55 Corner L, Bond J: The impact of the label of mild cognitive impairment on the individual's sense of self. Philos Psychiatr Psychol 2006;13:3-12.

56 Dale W, Hougham GW, Hill EK, Sachs GA: High interest in screening and treatment for mild cognitive impairment in older adults: a pilot study. J Am Geriatr Soc 2006;54:13881394

-57 Banningh LJ, Vernooij-Dassen M, Rikkert MO, Teunisse J: Mild cognitive impairment: coping with an uncertain label. Int J Geriatr Psychiatry 2008;23:148-154. 
58 Alladi S, Arnold R, Mitchell J, Nestor PJ, Hodges JR: Mild cognitive impairment: applicability of research criteria in a memory clinic and characterization of cognitive profile. Psychol Med 2006;36:507-515.

59 Thompson SA, Hodges JR: Mild cognitive impairment: a clinically useful but currently ill-defined concept? Neurocase 2002;8:405410.

60 Gilewski MJ, Zelinski EM, Schaie KW: The Memory Functioning Questionnaire for assessment of memory complaints in adulthood and old age. Psychol Aging 1990;5: 482-490.

61 Dixon RA, Hultsch DF, Hertzog C: The Metamemory in Adulthood (MIA) questionnaire. Psychopharmacol Bull 1988;24: 671-688.

-62 Lachman ME, Baltes PB, Nesselroade JR, Willis SL: Examination of personality-ability relationships in the elderly: the role of the contextual (interface) assessment mode. J Res Pers 1982;16:485-501.
63 Jorm AF, Jacomb PA: The Informant Questionnaire on Cognitive Decline in the Elderly (IQCODE): socio-demographic correlates, reliability, validity and some norms. Psychol Med 1989;19:1015-1022.

64 Kazui H, Watamori TS, Honda R: The validation of a Japanese version of the Everyday Memory Checklist (Japanese). No To Shinkei 2003;55:317-325.

65 Sehulster JR: Structure and pragmatics of a self-theory of memory. Mem Cognit 1981;9: 263-276.

66 Crook TH, Feher EP, Larrabee GJ: Assessment of memory complaint in age associated memory impairment: the MAC-Q. Int Psychogeriatr 1992;4:165-175.

67 Marson DC: Loss of financial competency in dementia: conceptual and empirical approaches. Aging Neuropsychol Cogn 2001;8: 164-181.

68 Marson DC, Sawrie SM, Snyder S, McInturff B, Stalvey T, Boothe A, Aldridge T, Chatterjee A, Harrell LE: Assessing financial capacity in patients with Alzheimer disease: a conceptual model and prototype instrument. Arch Neurol 2000;27:877-884.

-69 Marin RS, Biedrzycki RC, Firinciogullari S Reliability and validity of the Apathy Evaluation Scale. Psychiatry Res 1991;38:143162.
70 Pfeffer RI, Kurosaki TT, Harrah CH Jr, Chance JM, Filos S: Measurement of functional activities in older adults in the community. J Gerontol 1982;37:323-329.

7 Lawton MP, Brody EM: Assessment of older people: self-maintaining and instrumental activities of daily living. Gerontologist 1969; 9:179-186.

72 Squire LR, Zouzounis JA: Self-ratings of memory dysfunction: different findings in depression and amnesia. J Clin Exp Neuropsychol 1988;10:727-738.

73 Winblad B, Palmer K, Kivipelto M, Jelic V, Fratiglioni L, Wahlund LO, Nordberg A, Backman L, Albert M, Almkvist O, Arai H, Basun H, Blennow K, de Leon M, DeCarli C, Erkinjuntti T, Giacobini E, Graff C, Hardy J, Jack C, Jorm A, Ritchie K, van Duijn C, Visser P, Petersen RC: Mild cognitive impairment - beyond controversies, towards a consensus: report of the international working group on mild cognitive impairment. J Intern Med 2004;256:240-246. 